\title{
Molecular geometry and molecular graphics: Natta's polypropylene and beyond
}

\author{
Guido Raos \\ Dip. di Chimica, Materiali e Ing. Chimica "G. Natta", Politecnico di Milano \\ Via L. Mancinelli 7, 20131 Milano, Italy \\ guido.raos@polimi.it
}

\begin{abstract}
In this introductory lecture I will try to summarize Natta's contribution to chemistry and materials science. The research by his group, which earned him the Noble prize in 1963, provided unprecedented control over the synthesis of macromolecules with well-defined three-dimensional structures. I will emphasize how this structure is the key for the properties of these materials, or for that matter for any molecular object. More generally, I will put Natta's research in a historical context, by discussing the pervasive importance of molecular geometry in chemistry, from the 19th century up to the present day. Advances in molecular graphics, alongside those in experimental and computational methods, are allowing chemists, materials scientists and biologists to appreciate the structure and properties of ever more complex materials.
\end{abstract}

Keywords: molecular geometry, stereochemistry, chirality, polymers, self-assembly, Giulio Natta

To be presented at the 18th International Conference on Geometry and Graphics, Politecnico di Milano, August 2018: http://www.icgg2018.polimi.it/

\section{Introduction: the birth of stereochemistry}

Modern chemistry was born in the years spanning the transition from the 18th to the 19th century. Two key figures were Antoine Lavoisier (1943-1794), whose emphasis on quantitative measurements helped to transform alchemy into a science on an equal footing with physics, and John Dalton (1766-1844), whose atomic theory provided a simple rationalization for the way chemical elements combine with each other to form compounds. Figure 1 shows the graphical representation adopted by Dalton to describe a few common elements and compounds. Today we can easily spot some mistakes in his diagrams. For example, it did not occur to Dalton that identical atoms could bind to each other to form molecules (as in $\mathrm{H}_{2}$ ), and we all know that water is $\mathrm{H}_{2} \mathrm{O}$ rather than $\mathrm{HO}$. Nonetheless, these diagrams also convey some simple and powerful ideas-perhaps we should say "intuition", given the lack of hard evidence available at the time - which are still valid today. In particular, atoms are essentially spherical objects which may 
bind to each other to form larger aggregates (molecules, in today's wording), depending on their relative affinities and following some well-defined geometrical patterns.

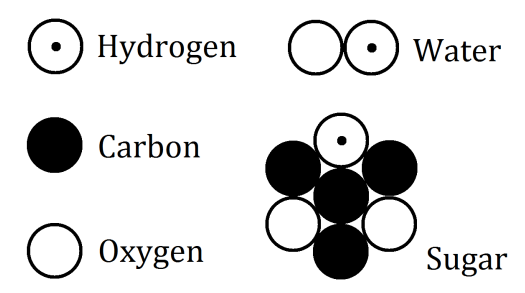

Fig. 1. Representation of atoms/elements and molecules/compounds according to Dalton (ca. 1808).

In the following years, chemists continously improved their experimental methods, allowing the discovery of new elements and the determination of the composition of many chemical substances. Above all, they refined their conceptual models of the internal structure of molecules and used them with increasing confidence in order to interpret experimental results. It is striking that this happened more than fifty years before molecular structures could be directly determined by X-ray diffraction or other methods, and chemical bonding interpreted by quantum mechanical principles (1910-1930).

In 1848, the young Louis Pasteur (1822-1895) observed the existence of two variants of the compound tartaric acid $\left(\mathrm{C}_{4} \mathrm{H}_{6} \mathrm{O}_{6}\right)$. The two forms had virtually identical chemical and physical properties, except for the fact that their solutions in water would interact differently with a beam of linearly polarized light passing through them. One would rotate to the right its plane of polarization, the other would rotate it to the left by exactly the same amount (at equal concentrations). The two versions or "optical isomers" of the compound were thus called D (from the Latin dextro, right-handed) and L (from the Latin laevo, left-handed). Indeed, it was believed that these two should be related in a way similar to our right and left hands (non-superposable mirror images of each other), and for this reason compounds with this property were called "chiral" (after the Greek $\chi \epsilon i \rho$, hand). The phenomenon of chirality was observed in many other natural compounds. In fact, nature seemed to have a "monopoly" in its ability to produce substances with a well-defined chirality (i.e., 100\% right- or left-handed). Instead, synthetic methods available at the time allowed chemists to produce only non-chiral compounds, or random mixtures of compounds with opposite chiralities (i.e., $50 \%$ right- and $50 \%$ left-handed).

The correct explanation for the origin of molecular chirality was given about twenty years later by Jacobus van't Hoff (1852-1911). By then it has been established that the C atom is "tetravalent", as it always forms four chemical bonds (some of these valences may be grouped to form multiple bonds, as in 


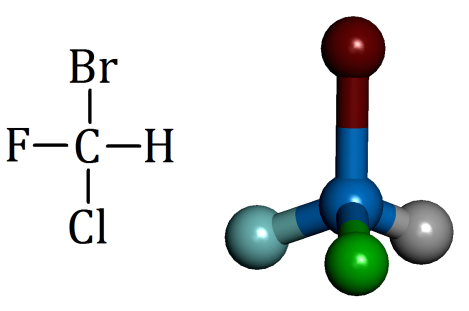

Fig. 2. Two-dimensional (misleading) and three-dimensional representations of the CHFClBr molecule.

carbon dioxide: $\mathrm{O}=\mathrm{C}=\mathrm{O}$ ). Van't Hoff understood that a situation with two nonsuperposable mirror images arises whenever a $\mathrm{C}$ atom is bonded to four different atoms or molecular fragments, but to do so those atoms must adopt a tetrahedral configuration (right-hand side of Fig.2). On the other hand, a two-dimensional model of the same or any other molecule (left-hand side of Fig.2) could never explain the existence of chirality, as a flat object has always left-right mirror symmetry. For this and other seminal contributions, Van't Hoff was awarded the first Nobel Prize in Chemistry in 1901.

The work of Pasteur and van't Hoff taught chemists that it is essential to think of molecules as three-dimensional objects. From then onwards the field of "stereochemistry" progressed rapidly, producing some striking results. Consider for example glucose $\left(\mathrm{C}_{4} \mathrm{H}_{6} \mathrm{O}_{6}\right)$, which is shown in Figure 3. This has five asymmetric tetrahedral carbons, so that in principle there should be at least $2^{5}=32$ versions or isomers of this compound (16 pairs of optical isomers). Around 1888 Emil Fischer (1852-1919) succeded in determining the relative configurations of those five carbon atoms (as shown on the left-hand side of Figure 3) by carefully studying the products of its reactions and applying some purely logical reasoning. His work is still taught to every chemistry student and it was rewarded with the 1902 Nobel Prize in Chemistry. The representation in the middle of Figure 3 contains much more information than that available to Fischer, as it can be drawn only with a knowledge of the precise three-dimensional arrangement of the atoms (bond lengths, angles, etc.). Finally, the picture on the right-hand side shows the electrostatic potential of a molecule, plotted on its "Van der Waals surface". This requires a knowledge of the molecule's charge distribution, which depends both on the positions of the nuclei (i.e., the details of the molecular geometry) and the quantum-mechanical distribution of the electrons around them. This sort of representation becomes extremely important when one wants to understand not just the structure of a molecule, but also its weak "non-bonding" interactions with other molecules (e.g., when it crystallizes or dissolves in water).

We are now ready to introduce Natta's discovery of stereoregular polymers. Much of whas has been said so far and will be said in the next section can be found in the book by him and Mario Farina.[1] A copy of the Italian edition of the book can be downloaded from the Natta archives, alongside his whole scientific production and many other documents.[2] The web site of the Nobel 


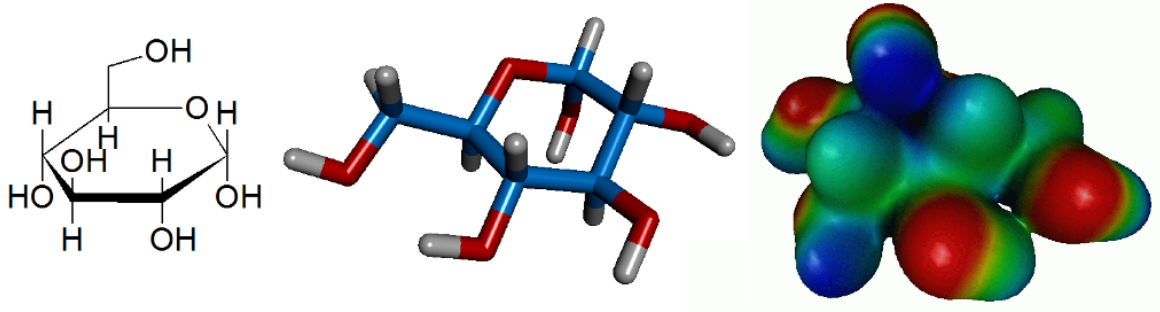

Fig. 3. Increasingly sophisticated representations of the three-dimensional structure of the glucose molecule. The were produced, from left to right, with ChemSketch[7], DS Visualizer[8] and MOLDEN [9]. The representation on the left emphasizes the positions of the dangling groups with respect to the six-membered ring, sacrificing for clarity the tetrahedral geometry of each carbon. The representation on the right displays the electrostatic potential at the surface of the molecule.

Foundation is also a good starting point to read about Natta, as well as the scientists which have been and will be mentioned.[3]

\section{Natta's polypropylene: the breaking of Nature's monopoly}

Giulio Natta (1903-1979) was a brilliant student. He graduated in his early twenties in chemical engineering, rose quickly through the academic ranks and was appointed Professor of industrial chemistry at the Politecnico di Milano in 1938. His research interests were extremely broad, ranging from the determination of crystal structures to the development of chemical processes for the petroleum industry.

In the early 1950's Natta became aware of Karl Ziegler's advances in the development of catalysts for the polymerization of ethylene $\left(\mathrm{CH}_{2}=\mathrm{CH}_{2}\right)$. The product of the reaction is polyethylene, which consists of very long molecular chains (i.e., $\left(-\mathrm{CH}_{2}-\mathrm{CH}_{2}-\right)_{n}$, where $\left.n>10^{3}\right)$. With the industrial backing of Montecatini, Natta started a strong research program involving the application of similar catalysts to the polymerization of other molecules, including propylene $\left(\mathrm{CH}_{2}=\mathrm{CH}-\mathrm{CH}_{3}\right)$. In 1954 his group succeeded in the sythesis and structural chacterization of isotactic polypropylene (iPP), which since then has become one of the most widespread and versatile commodity plastics (see Figure 4).

Since the very beginning, Natta realized that the favourable properties of iPP (e.g., mechanical rigidity and high melting point) were linked to its crystallinity. In turn, this depends on the precise arrangement of the $-\mathrm{CH}_{3}$ groups dangling from the main chain. They are all on the same side of it, and this allows the chains to adopt a regular helical geometry and pack very efficiently in the solid state. Note in passing that helices are also chiral, as they can be either left- or righthanded. Using a differerent polymerization catalyst, it was also possible to make syndiotactic polypropylene ( $\mathrm{sPP}$ ), in which the main groups alternate between 

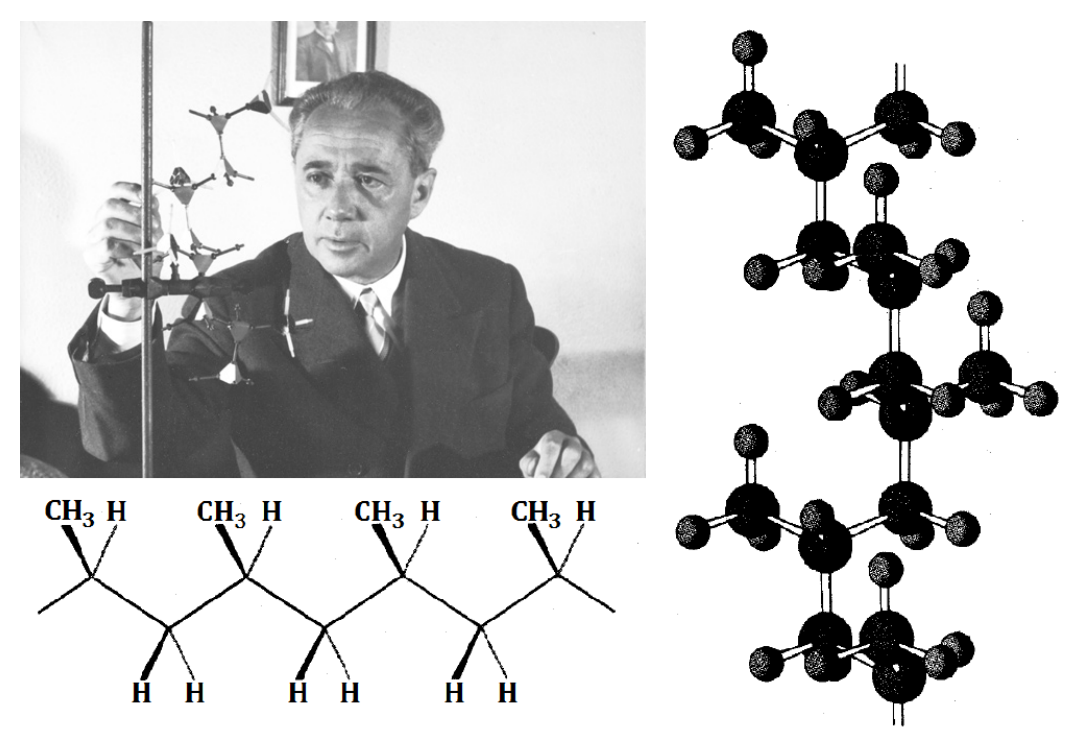

Fig. 4. From top left, counterclockwise: Natta contemplating a three-dimensional model of isotactic polypropylene (iPP), a representation of iPP in an hypothetical (high-energy) zig-zag conformation, and a representation of the actual (low-energy) helical conformation adopted by iPP in the crystalline state. The zig-zag and helical conformations differ by the dihedral angles formed by C-C-C-C quadruplets along the chain backbone. Pictures taken and adapted from refs.[1] and [2]. 
opposite sides of the main chain. In 1963 Natta was awared the Nobel Prize in Chemistry (shared with Ziegler), for having succeeded in breaking nature's monopoly in the synthesis of macromolecules with precisely controlled structures.

It is interesting that Natta's work on synthetic polymers was almost simultaneous with other history-making discoveries about the structure of natural macromolecules. Interestingly, also these frequently adopt a helical pattern: think of the $\alpha$-helical motif in proteins discovered by Linus Pauling and coworkers (see Figure 5 for an example involving Hemoglobin) and of DNAs double helix discovered by James Watson and Francis Crick. Clearly, the building of three-dimensional physical models and the accurate graphical representation of molecular structures were central to these exciting discoveries.

\section{Outlook: structure and self-assembly in biology and materials}

In the fifty years since the pioneering work of Natta and his contemporaries, our understanding of the structure and properties of both small and large molecules has grown enormously. There has been also a tremendous increase in chemists' ability to make new materials with intricate but well-defined structures. It is impossible to summarize the main developments within the limited space of this contribution. Therefore I will simply fast-forward and offer a couple of examples of what has been achieved in the last few years. One key theme which connotates many research advances is the investigation of "supra-molecular" systems. These are obtained when two or more molecules with complementary interactions organize into more complex structures. These "self-assembly" processes do not involve the breaking or formation of chemical bonds, but they rely on a delicate balance of a large number of weaker, non-bonding interactions. $\mathrm{Hu}$ man Hemoglobin is one example as it is formed by aggregation of four protein subunits (see the right-hand side of Figure 5).

The development of cryo-electron microscopy is an example of truly revolutionary progress in methods for structural determination, especially for large biomolecular assemblies that cannot be easily crystallized (this prevents the application of more conventional X-ray diffraction methods). Jacques Dubochet, Joachim Frank and Richard Henderson were awarded the 2017 Nobel Prize in Chemistry for its development. The investigation of complex molecular structures and their properties now relies very much on the application computational approaches, or "molecular modelling". These were pioneered, among others, by Martin Karplus, Michael Levitt and Arieh Warshel, who received the Nobel Prize in 2013. As an example of a structure obtained by a combination of cryo-electron microscopy and molecular simulations, I mention the capsid of the HIV-1 virus.[4] This is made up by self-assembly of the capsid proteins into 216 hexamers (brown) and 12 pentamers (green), and these in turn assemble to form a shell enclosing the virus' genetic materials. The whole structure could be described as a higher fullerene (the first in the series being $\mathrm{C}_{60}$ ), in which however the place of each $\mathrm{C}$ atom is taken by a whole protein. The overall structure 

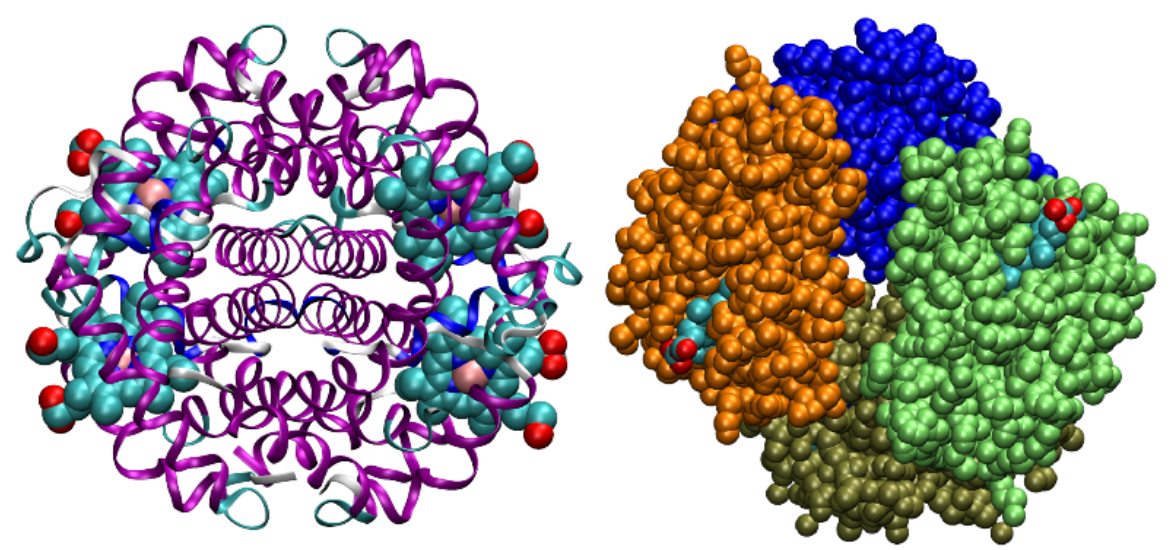

Fig. 5. The structure of human Hemoglobin. On the left-hand side, the $\alpha$-helical elements are shown in purple, using a "ribbon" representation. On the right-hand side, the four polypetide chains making up Hemoglobin are colored differently, using a spacefilling representation. In both cases, the four iron-containing, oxygen-binding "heme" groups are shown with a space-filling representation. The structure of Hemoglobin was first determined by Max Perutz and John Kendrew (Nobel Prize in Chemistry in 1962). Pictures produced with the VMD program,[10] using atomic coordinate downloaded from the Protein Data Bank.[11]

has a size exceeding $60 \mathrm{~nm}$ and contains 13 million atoms! Of course, the capsid not only self-assembles but may also disassemble to release the genetic material enclosed within it, in response to changes in the physico-chemical conditions after the virus enters a cell and infects it.

The self-assembly of proteins and other biomolecules into intriguing, often highly symmetrical structures has several parallels in the field of artificial materials. I will mention here the highly porous solids and molecular cages which can be obtained by "mixing" suitable metal ions and bi- or multi-functional organic molecules capable of establishing rigid bridges between them. Many of these systems go under the name of metal-organic frameworks (MOFs), and they are good examples of the so-called bottom-up approach to the production of nanomaterials. Such systems may find applications as molecular containers capable of trapping or releasing host molecules in a controlled way (e.g. hydrogen, carbon dioxide or water, but also much larger molecules such as pharmaceuticals or pollutants). To many chemists, however, they also have a purely aesthetic appeal. For example, Makoto Fujita and his group have synthesized near-spherical (actually, icosidodecahedral) supra-molecular cavities with a diameter of $8 \mathrm{~nm}$ (i.e., comparable to the size of Hemoglobin) consisting of $30 \mathrm{Pd}^{2+}$ ions and 60 banana-like molecules linking them.[5] He and Omar Yaghi shared the 2018 Wolf Prize in chemistry for the development of these materials. [6]

In conclusion, molecular geometry has always played a very important role in chemistry, and for very good reasons. It should also be clear that the investi- 
gation of ever more complex structures has stimulated and benefitted from the development of increasingly sophisticated graphical representations. In fact, virtual reality technologies are already being used in some laboratories in order to appreciate complex chemical systems, and their use is certainly going to expand even further in the next few years. Natta would be pleased and excited by these developments.

Acknowledgements. I wish to thank Luigi Cocchiarella for the invitation to give this introductory lecture. I also thank Renato Rota for the kind encouragement and Stefano Valdo Meille for helpful discussions.

\section{References}

1. Natta, G., Farina, M.: Stereochimica. Molecole in 3D. Mondadori, Milano (1968). English translation: Stereochemistry. Harper and Row, New York (1973).

2. The Giulio Natta archive. http://www.giulionatta.it/

3. The Nobel Prizes in Chemistry. https://www.nobelprize.org/nobel_prizes/chemistry/laureates/

4. Zhao, G., Perilla, J. R., Yufenyuy, E. L., Meng, X., Chen, B., Ning, J., Ahn, J., Gronenborn, A. M., Schulten, K., Aiken, C., Zhang, P.: Mature HIV-1 capsid structure by cryo-electron microscopy and all-atom molecular dynamics. Nature 497, 643-646 (2013). doi:10.1038/nature12162

5. Fujita, D., Ueda, Y., Sato, S., Yokoyama, H., Mizuno, N., Kumasaka, T., Fujita, M.: Self-Assembly of $\mathrm{M}_{30} \mathrm{~L}_{60}$ Icosidodecahedron. Chem 1, 91-101 (2016). doi:10. 1016/j.chempr.2016.06.007

6. The Wolf foundation. http://www.wolffund.org.il/

7. ACD/Labs ChemSketch. http://www.acdlabs.com/

8. BIOVIA DS Visualizer. http://accelrys.com/

9. MOLDEN. http://www.cmbi.ru.nl/molden/

10. Humphrey, W., Dalke, A., Schulten, K.: VMD - Visual Molecular Dynamics. J. Molec. Graphics 14, 33-38 (1996). https://www-s.ks.uiuc.edu/Research/vmd/

11. The Protein Data Bank. https://www.rcsb.org/ 